\title{
Impact of the Severity of Liver Injury in COVID-19 Patients Admitted to an Intensive Care Unit During the SARS-CoV2 Pandemic Outbreak
}

\author{
Adina Roman ${ }^{1,2,3}$, Septimiu Moldovan2* , Ario Santini2, Mircea Stoian ${ }^{1,2}$, Daniela Dobru ${ }^{1,2}$ \\ 1 Mures County Hospital, Targu Mures, Romania \\ 2 George Emil Palade University of Medicine, Pharmacy, Science, and Technology of Targu Mures, Romania \\ 3 Doctoral School, George Emil Palade University of Medicine, Pharmacy, Science, and Technology of Targu Mures, \\ Romania
}

\begin{abstract}
Introduction: The World Health Organization (WHO) identified a novel coronavirus, originating in Wuhan, China, in December 2019, as a pneumonia causing pathogen. Epidemiological data in Romania show more than 450.000 confirmed patients, with a constant number of approximately $10 \%$ admission in intensive care unit. Method: A retrospective, observational study was conducted from 1st March to 30th October 2020, comprising 657 patients, confirmed as having COVID-19, and who had been admitted to the intensive care unit of the Mures County Clinical Hospital, Tîrgu Mures, Romania, which had been designated as a support hospital during the pandemic. Patients who presented at admission or developed abnormal liver function tests in the first seven days of admission, were included in the study; patients with pre-existing liver disease, were excluded. Results: The mean (SD) age of patients included in the study was 59.41 (14.66) years with a male: female ratio of 1.51:1. Survivor status, defined as patients discharged from the intensive care unit, was significantly associated with parameters such as age, leukocyte count, albumin level, glycaemia level ( $p<0.05$ for all parameters.) Conclusions: Liver injury expressed through liver function tests cannot solely constitute a prognostic factor for COVID-19 patients, but its presence in critically ill patients should be further investigated and included in future guideline protocols.
\end{abstract}

Keywords: COVID-19, liver injury, SARS-COV2, pandemic outbreak

Received: 30 November 2020 / Accepted: 27 June 2021

\section{INTRODUCTION}

The World Health Organization (WHO) identified a novel coronavirus originating in Wuhan, China in December 2019, as a pathogen which causes pneumonia [1].

The novel coronavirus, officially named SARSCoV-2, and the disease caused by it, COVID-19, spread quickly in more than 60 countries [2].

More than nine months after the diagnosis of the first patient with SARS-CoV-2 infection, epidemiological data in Romania accounted for more than 450.000 confirmed patients, with the total number of deaths surpassing 11.000 cases (2.37\%). Approximately $10 \%$ of all patients confirmed as having the virus, required to be admitted to an intensive care unit [3].

SARS-COV2 infection is already known to be transmitted through respiratory droplets and manifests through variate symptoms such as fever, difficulty breathing, dry cough, and most recently, with a switch towards abdominal symptoms: pain, diarrhoea, nausea, or vomiting [4].

Many studies have tried to clarify the association between liver function tests and COVID-19, validating hypotheses such as drug-induced liver injury or direct hepatocyte damage due to increased viral load $[5,6]$.

Liver function tests in patients with COVID-19 showed the liver to be the most frequently damaged organ, with the exception of the respiratory system [7].

SARS-CoV2 related liver injury have been defined as a hepatocellular pattern, cholestatic or mixed patterns. (Table 1.) Garcia-Cortes et al (2020) classified COVID-19 liver injury as mild, moderate or severe according to liver function tests (Table 2.) 
Table 1. Classification of the pattern of liver injury.

Hepatocellular pattern: Alanine transaminase levels $\geq 5$ upper limit of normal; alanine transaminase levels/alkaline phosphatase levels $\geq 5$.

Cholestatic pattern: Alanine transaminase levels $\geq 2$ upper limit of normal; alanine transaminase levels /alkaline phosphatase $\leq 2$.

Mixed pattern: Alanine transaminase levels/alkaline phosphatase 2-5.

Table 2. Forms of SARS-COV2 associated liver disease

A mild form of the disease: Mild symptoms without clinical or para clinical signs of pneumonia, or with a Chest X-Ray or CT scan showing $<25 \%$ parenchymal damage.

A moderate form of the disease: Mild to moderate symptoms with para clinical signs of pneumonia, or a Chest X-Ray or CT scan showing a $25-50 \%$ parenchymal damage.

A severe form of the disease: With a respiratory rate $\geq 30 / \mathrm{min}, \mathrm{pSO2} \leq 93 \%, \mathrm{PaO} 2 / \mathrm{FiO} 2 \leq 300 \mathrm{~mm} \mathrm{Hg}$, or a Chest X-Ray or CT scan showing more than $50 \%$ parenchymal damage, or signs of acute respiratory distress, confusion/coma, sepsis, shock.

However, in their meta-analysis, Wu et al. (2016) drew attention to the similarity between the mild and moderate groups of COVID-19 liver injury. Their opinion was that considering the small clinical differences between the mild and moderate groups, as well as their possible impact on patients' outcome, the two groups should be combined and further suggested that consideration should be given to updating the guidelines to uphold this view.

The current study aimed to establish whether the severity of liver injury is a supplementary outcome prognostic factor in COVID-19 patients admitted to an intensive care unit. The null hypothesis is that the severity of liver injury is not a supplementary outcome prognostic factor in COVID-19.

\section{- METHODS}

A retrospective, observational study was conducted from $1^{\text {st }}$ March to $30^{\text {th }}$ October 2020, on patients, confirmed as having COVID-19, and who had been admitted to the intensive care unit of the Mures County Clinical Hospital, Tîrgu Mures, Romania, which had been designated as a support hospital during the pandemic.

Inclusion Criteria: Adult patients who, on admission to the intensive care unit, presented with abnormal liver function tests and laboratory confirmation of COVID-19 infection i.e. detection of SARS-COV2 RNA in a clinical specimen using a molecular amplification detection test, or developed abnormal liver function tests with and laboratory confirmation of COVID-19 infection i.e. detection of SARS-COV2 RNA in a clinical specimen using a molecular amplification detection test, in the first seven days of admission.
Exclusion Criteria: Adult patients with a history of any type of pre-existing liver disease.

Case Notes contained: Demographics: age, sex, were recorded on admission, as were the following associated comorbidities ischemic heart disease, diabetes mellitus, haematological conditions, active cancer, as well as history of cancer, metastatic disease, neurological and rheumatological conditions.

Clinical outcomes such as death, and length of stay in the intensive care unit were recorder during the stay in the ICU for future analysis.

Laboratory tests conducted on the day of admission, including a complete blood count (haemoglobin, platelet count, and leukocyte count), renal function tests (urea, creatinine), electrolyte count (sodium, potassium), biochemical tests (glycaemia, albumin, cholesterol, and triglycerides), and inflammatory response markers (CRP, Ferritin and erythrocyte sedimentation rate).

All medications administered during the stay in the intensive care unit.

Appraisal of the pattern of liver injury was characterized as hepatocellular pattern, cholestatic or mixed patterns, according to Andrade et al. (2019) [8] Table 1.

SARS-COV2 related liver injury was defined as liver injury detected and confirmed by abnormal liver biochemical and function tests including alanine aminotransferase, aspartate aminotransferase, alkaline phosphatase, gamma-glutamyl transferase, in patients with confirmed evidence of SARS-CoV2 infection. Table 3 .

Patients were allocated to two groups for further analyses. 
Table 3. Classification of liver injury

Mild: Liver function tests 3-5 upper limit of normal.

Moderate: Liver function tests 5-10 upper limit of normal.

Severe: Liver function tests $\geq 10$

Group 1. Liver function tests 3-10 upper limit of normal. (This combined patients with both mild and moderate liver injury).

Group 2. Liver function tests $>10$ upper limit of normal (This comprised patients with severe liver injury.)

Comparisons were made between Group 1 and Group 2 using Student's t-tests or the Mann-Whitney U-test. Clinical outcomes, such as length of intensive care unit stay or death for each of the two groups were compared using Student's t test and Fisher's exact test. Associated comorbidities of the patients were evaluated using the Charlson Comorbidity Index.

Univariable and multivariable logistic regression models were used to establish additional risk factors for liver injury. Statistical tests were performed using SPSS19.0 software.

The level of significance was set at $\alpha=0.05$ for all tests.

\section{RESULTS}

From a total of 657 patients admitted to the intensive care unit, 156 patients (23.74\%) who presented with abnormal liver function tests on admission or during the first seven days of hospitalisation were included in the study. The mean (SD) age was 59.41 (14.66) years with a male: female ratio of 1.5:1.

There was a significant difference between Groups 1 and 2 with respect to platelet count leukocyte count, urea, glycaemia, triglycerides, ferritin values, but not haemoglobin, creatinine, sodium or potassium values.

There was no statistical difference between the two groups with respect to recorded deaths but a significant difference with respect to the length of stay in hospital (Student $\mathrm{t}$ test $\mathrm{p}=0.009$ ). The null hypothesis is therefore partly upheld. Table 4 .

The following liver diseases were recorded in the study cohort: chronic viral hepatitis, liver cirrhosis, alcoholic liver disease, autoimmune hepatitis, malignancy or metastatic disease.

During hospitalisation, thirty-five patients (22.43\%) presented with mild liver injury, sixty-three (40.38\%) moderate liver injury, and fifty-eight (37.19\%) presented with severe liver injury.

Table 4. Demographics and para clinical characteristics of 156 enrolled COVID-19 patients.

\begin{tabular}{|c|c|c|c|}
\hline & Group 1. (n=98) & Group 2. $(n=58)$ & p-value \\
\hline \multicolumn{4}{|l|}{ Complete blood count } \\
\hline Haemoglobin $(\mathrm{g} / \mathrm{dL})$ mean $(\mathrm{SD})$ & $13.74(1.5)$ & $13.32(2.65)$ & Student $t$ test $p=0.377$ \\
\hline Platelet count $\left(10^{3} / \mathrm{uL}\right)$ mean $(\mathrm{SD})$ & $212.71(80.32)$ & $265.77(13.05)$ & Student $\mathrm{t}$ test $\mathrm{p}=0.035 *$ \\
\hline Leukocyte count (/uL) mean (SD) & $6310.69(2603.41)$ & $8867.69(5205.24)$ & Student $\mathrm{t}$ test $\mathrm{p}=0.003^{*}$ \\
\hline \multicolumn{4}{|l|}{ Renal function tests } \\
\hline Urea (mg/dL) mean (SD) & $36.49(15.36)$ & $57.66(23.59)$ & Student $\mathrm{t}$ test $\mathrm{p}=0.007 *$ \\
\hline Creatinine (mg/dL) mean (SD) & $0.85(0.21)$ & $0.89(0.24)$ & Student t test $p=0.592$ \\
\hline \multicolumn{4}{|l|}{ Electrolyte count } \\
\hline Sodium(mmol/L) mean (SD) & $138.73(3.43)$ & $137.15(4.88)$ & Student $t$ test $p=0.135$ \\
\hline Potassium(mmol/L) mean (SD) & $4.33(0.5)$ & $4.41(0.74)$ & Student $t$ test $p=0.603$ \\
\hline \multicolumn{4}{|l|}{ Biochemical tests } \\
\hline Glycaemia (mg/dL) mean (SD) & 118.59 (51.92) & $166.23(87.71)$ & Student $\mathrm{t}$ test $\mathrm{p}=0.004 *$ \\
\hline Albumin $(\mathrm{g} / \mathrm{L})$ mean $(\mathrm{SD})$ & $3.11(0.36)$ & $3.22(0.73)$ & Student $t$ test $p=0.574$ \\
\hline Cholesterol (mg/dL) mean (SD) & 182.16(49.27) & $200.43(46.17)$ & Student $t$ test $p=0.364$ \\
\hline Triglycerides (mg/dL) mean (SD) & 139(70.79) & $247.29(171.41)$ & Student $t$ test $p=0.005$ \\
\hline \multicolumn{4}{|l|}{ Inflammatory response markers } \\
\hline $\mathrm{CRP}(\mathrm{mg} / \mathrm{dL})$ mean $(\mathrm{SD})$ & $4.7(5.47)$ & $7.81(7.93)$ & Student $t$ test $p=0.065$ \\
\hline Ferritin (ng/mL) mean (SD) & $929.17(982.6)$ & $2597.677(2233.74)$ & Student $\mathrm{t}$ test $\mathrm{p}=0.02 *$ \\
\hline \multicolumn{4}{|l|}{ ICU } \\
\hline Length of stay in ICU. mean (SD) & $13.85(9.52)$ & $8.05(5.43)$ & Student $\mathrm{t}$ test $\mathrm{p}=0.009 *$ \\
\hline Death $(\mathrm{Y} / \mathrm{N})$ & $26 / 72$ & $35 / 23$ & Mann Whitney $U$ test $p=0.57$ \\
\hline
\end{tabular}


The median duration of liver injury was six days irrespective of severity of liver injury.

At the time of discharge, no patients exhibited normal liver function tests, regardless of the severity of their initial liver injury or their length stay in the intensive care unit.

There was a significant statistical difference between Groups 1 and 2 with respect to age, platelet and leukocytes count, urea, and ferritin level together with the length of admission in the intensive care unit (Student $\mathrm{t}$ test, $\mathrm{p}<0.05$ ) as seen in Table 4.

Survivor status, as defined as discharged patients from intensive care unit, was significantly associated with age, leukocyte count, albumin level, glycaemia level. $(\mathrm{p}<0.05)$

Comparison was made between patients in Groups 1 and 2. Patients in Group 2 were older (Pearson correlation parametric test; $\mathrm{p}=0.03$ ) and had a lower platelet count (Pearson correlation parametric test; $\mathrm{p}=0.02$ ) or leukocyte count (Pearson correlation parametric test; $\mathrm{p}=0.043$ ) and higher glycaemic (Pearson correlation parametric test; $\mathrm{p}=0.01$ ) and triglycerides levels (Pearson correlation parametric test; $\mathrm{p}=0.025$ ).

The pattern of SARS-COV2 related liver injury was assessed; there were no significant statistical differences between Groups 1 and Group 2 regarding hepatocellular (Fisher's two tailed exact test; $\mathrm{p}=0.23$ ), cholestatic (Fisher's two tailed exact test; $\mathrm{p}=0.83$ ) or mixed pattern (Fisher's two tailed exact test; $\mathrm{p}=0.20$ ). For both groups, hepatocellular injury was the predominant pathogenic mechanism (Group 1 - 55.1\%, Group 2 - 65.5\%).

The Charlson Comorbidity Index score had a value lower 2 points in 119 patients (76.2\%), associated with more than $90 \%$ estimated 10 -year survival rate.

The use of glucocorticoids, antibiotic therapy, antiviral therapy, acetaminophen in patients in Group 1 were not associated with a risk of additional liver injury (Fisher's exact test; $p>0.05$ ). In Group 2, the use of antifungal therapy was associated with a low risk for an additional liver injury - OR 1.04 (95\% CI [0.95-1.14]).

An abdominal computed tomography scan was performed on patients with severe liver injury (37.19\%) and there were no records of intrahepatic or extrahepatic obstruction, liver cirrhosis or hepatomegaly, suggestive of pre-existing liver disease.

Complete etiological investigation of liver injury was performed in only six patients (3.8\%).

\section{DISCUSSION}

The search for diagnostic and prognostic biomarkers in patients in severely ill patients admitted to an intensive care unit, at the time of admission, is a medical challenge when dealing with unknown issues of SARSCOV2 infection $[9,10]$.

The present study comprises an accurate descriptive statistics of SARS-COV2 patients with abnormal liver function admitted to intensive care unit. The data demonstrated that the severity of liver injury is not a supplementary outcome prognostic factor and highlighted the significance of paraclinical follow-up in SARS-COV2 patients.

The current pathogenic mechanisms involved in multi-organ injury in SARS-COV2 related secondary infections include direct toxicity, endothelial cell damage and thrombo-inflammation [11,12], dysregulation of the immune response and the renin-angiotensinaldosterone system. These are suspected to contribute to a direct effect on the liver and be associated with gastrointestinal symptoms $[13,14]$.

Similar to the results of the current study, there has been confirmation that elevated liver function tests are more common in patients with a severe type of COVID-19, most likely due to association with inflammatory cytokine storm $[15,16]$.

In most cases, function abnormalities, regardless of the predominant action mechanism were limited to duration [15]. Consistent with previous studies $[17,18]$, the present study found that severe COVID-19 patients presented early liver injury onset and a slow decrease of in abnormality of liver function tests, with a lack of normalisation of liver function tests at the time of discharge.

More studies are required to establish the pathogenesis of liver function tests abnormalities in patients with mild SARS-CoV-2 infection admitted to intensive care unit for associated severe comorbidities.

In a systematic review, Parohan et al. (2020) analysed twenty retrospective studies focused on liver injury in COVID-19 and correlated serum levels of aspartate aminotransferase, alanine transaminase, total bilirubin and albumin, with the severity of COVID-19 infections [19]. Parohan's meta-analysis underlined the current consensus, which rules out the additional hepatotoxic effect of drug combinations, including, but not limited to, steroids, antiviral, antibiotics or antimycotic treatment. 
The present study corroborates this report; the only correlation which was not be established was between the level of albumin or total bilirubin with the severity of COVID-19.

Lopinavir-ritonavir treatment was believed to induce liver dysfunction, but more recent studies ruled out this hypothesis, maintaining that a key treatment consideration is monitoring hepatotoxicity of the administered medications [20].

Jiang et al.(2020) in a multicentre, retrospective observational study, compared liver injury in critically ill with non-critically ill patients, concluding that drug factors were independent risk factors for non-critically ill patients, and drug interactions of concomitant drugs should be closely monitored, especially in this group of patients [21]. The present study established the correlation between liver injury and the use of antifungal therapy in severe-COVID19 patients admitted to intensive care unit.

The Romanian Society of Anaesthesia \& Intensive Care published a Romanian guideline for critically ill patient with COVID-19 in intensive care units Departments. One crucial aspect discussed in the guidelines is managing patients with acute liver failure associated with COVID-19. Previous reports state that the number of patients who required admission to an intensive care unit due to acute liver failure is relatively small though the mortality rate in these patients was very high. The primary guideline recommendations for these patients include the limitation of usage of hepatotoxic drugs, checking the availability for transfer in specialised centres, using the antiviral drugs approved by the national health organisation. Listing for hepatic transplant, which can be controversial, is also included [22]. Consultant hepatologists should base their multidisciplinary approach, together with intensive care physicians, by increasing the rate of performing aetiological and imagistic investigations in patients with SARS-COV2 liver injury [23].

Updated national and international guidelines of liver injury management in COVID-19 are required to aid in the rapid differential diagnosis and treatment of the disease.

Further studies are required to investigate the pathogenesis of COVID-19 liver injury, and the relation of causality between different pathogenetic triggers. Our study helps to emphasise the mandatory follow-up of abnormal liver function tests as well as establishing a proper management in critically-ill patients.

\section{- CONCLUSIONS}

SARS-COV2 infection has been associated with liver function impairment; abnormal liver function tests are common in COVID-19 patients and are associated with increased severity of the disease.

The pathogenesis of liver injury, especially in intensive care unit patients is multifactorial and requires further research. Drug-induced liver disease associated with antiviral drug use cannot be excluded as a pathophysiological mechanism, especially in the use of concomitant therapies in intensive care unit patients.

Liver injury expressed through liver function tests abnormality does not solely constitute a prognostic factor for COVID-19 patients, but its presence in critically ill patients should be suspected and investigated in all cases.

\section{AUTHORS' CONTRIBUTION}

AR - Conceptualisation, investigation, data analysis, methodology, writing the original draft

SM - data analysis, statistical analysis, corresponding author

MS - Reviewing and editing, data analysis

DD - Conceptualisation, investigation, re-viewing and editing the original draft

\section{CONFLICT OF INTEREST}

None to declare.

\section{REFERENCES}

1. Fan Z, Chen L, Li J et al. Clinical features of COVID-19-related liver functional abnormality. Clin. Gastroenterol. Hepatol 2020;18(7):1561-6.

2. https://www.who.int/dg/speeches/detail/who-directorgeneral-s-remarks-at-the-media-briefing-on-2019-ncov-on11-february-2020

3. http://www.ms.ro/coronavirus-covid-19/

4. https://www.worldometers.info/coronavirus/\#countries

5. Qingxian C, Deliang H, Hong Y, et al. COVID-19: abnormal liver function tests. J Hepatol. 2020; Sep: 566-74. doi:/10.1016/j. jhep.2020.04.006

6. Wang D, Hu B, Hu C,et al. Clinical characteristics of 138 hospitalized patients with 2019 novel coronavirus-infected pneumonia in Wuhan, China. JAMA.2020; 323 (11): 10611069. doi:10.1001/jama.2020.1585 
216 The Journal of Critical Care Medicine 2021;7(3)

7. Xiaolong $\mathrm{Q}$, Chuan $\mathrm{L}$, Jiang $\mathrm{Z}$, et al. Multicenter analysis of clinical characteristics and outcomes in patients with COVID-19 who develop liver injury. J Hepatol. 2020; 73(2): 455-458. doi: 10.1016/j.jhep.2020.04.010

8. Andrade RJ, Chalasani N, Bjornsson ES, et al. Drug-induced liver injury. Nature Reviews Disease Primers. 2019; 5(1): 1-22.

9. Chaolin $H$, Yeming $W$, Xingwang $L$, et al. Clinical features of patients infected with 2019 novel coronavirus in Wuhan, China.The Lancet. 2020; 395.10223: 497-506. https://doi. org/10.1016/S0140-6736(20)30183-5

10. Yang AP, Liu JP, Tao WQ, et al. The diagnostic and predictive role of NLR, d-NLR and PLR in COVID-19 patients. International immunopharmacology. 2020;106504. https://doi. org/10.1016/j.intimp.2020.106504

11. Henry BM, Santos de Oliveira MH, Benoit S, et al. Hematologic, biochemical and immune biomarker abnormalities associated with severe illness and mortality in coronavirus disease 2019 (COVID-19): a meta-analysis. Clinical Chemistry and Laboratory Medicine (CCLM). 2020; 58.7: 1021-1028. https://doi. org/10.1515/cclm-2020-0369

12. Yueying L, Xiao SY. Hepatic involvement in COVID-19 patients: pathology, pathogenesis and clinical implications. Journal of medical virology. 2020; https://doi.org/10.1002/jmv.25973

13. Wiersinga WJ, Rhodes A, Cheng CA, et al. Pathophysiology, transmission, diagnosis, and treatment of coronavirus disease 2019 (COVID-19): a review. Jama. 2020; 324.8: 782-793. doi:10.1001/jama.2020.12839

14. Gupta A, Mandhavan M, Landry D, et al. Extrapulmonary manifestations of COVID-19. Nature medicine. 2020; 26.7: 1017-1032. https://doi.org/10.1038/s41591-020-0968-3.

15. Bertolini A, Van de Peppel PI, Bodewes F et al. Abnormal liver function tests in COVID-19 patients: relevance and potential pathogenesis. Hepatology. 2020; 72(5):1864-1872 https://doi.
Available online at: www.jccm.ro

org/10.1002/hep.31480

16. Bloom PP, Trisha SP, Viveiros K. We know liver biochemistries are elevated in COVID-19, but should we be concerned? Clinical Gastroenterology and Hepatology. 2020; 18.10: 23842385. https://doi.org/10.1016/j.cgh.2020.05.018

17. Alqahtani SA, Jörn MS. Liver injury in COVID-19: The current evidence. United European Gastroenterology Journal. 2020; 8.5: 509-519. https://doi.org/10.1177/2050640620924157

18. Ridruejo E, Soza A. The liver in times of COVID-19: What hepatologists should know. Annals of Hepatology. 2020; 19(4):353-358

19. Parohan M, Sajad Y, Asal S. Liver injury is associated with severe Coronavirus disease 2019 (COVID-19) infection: a systematic review and meta-analysis of retrospective studies. Hepatology Research.2020; 50(8):924-935. https://doi.org/10.1111/ hepr.13510

20. Boettler T, Newsome PN, Mondelli MU, et al. Care of patients with liver disease during the COVID-19 pandemic: EASL-ESCMID position paper. JHEP Reports. 2020; 2(3):100113. https://doi. org/10.1016/j.jhepr.2020.100113

21. Jiang $S$, Wang R, Li L, et al. Liver Injury in Critically III and Noncritically III COVID-19 Patients: A Multicenter, Retrospective, Observational Study. Frontiers in medicine. 2020; 7:347. https://doi.org/10.3389/fmed.2020.00347

22. Bubenek S, Filipescu D, Grigoras I, et al. Ghid Societatea Romană de Anestezie și Terapie Intensivă - Terapia Intensivă a pacientului critic cu boală COVID-19 in secțiile ATI .2020. pp.3038

23. Zheng $Y$, Song B. COVID-19 related liver injury: call for international consensus. Clinical Gastroenterology and Hepatology. 2020; 18(12): 2848-2851. https://doi. org/10.1016/j.cgh.2020.05. 